\title{
BRCA1 Forms a Functional Complex with $\gamma$-H2AX as a Late Response to Genotoxic Stress
}

\author{
Susan A. Krum, ${ }^{1}$ Esther de la Rosa Dalugdugan, ${ }^{2}$ Gustavo A. Miranda-Carboni, ${ }^{2}$ \\ and Timothy F. Lane ${ }^{1,2,3}$ CA 90095, USA
${ }^{2}$ Department of Obstetrics and Gynecology, Jonsson Comprehensive Cancer Center, David Geffen School of Medicine at UCLA, Los Angeles, CA 90095, USA
${ }^{3}$ Department of Biological Chemistry, Jonsson Comprehensive Cancer Center, David Geffen School of Medicine at UCLA, Los Angeles, CA 90095, USA \\ ${ }^{1}$ Molecular Biology Institute, Jonsson Comprehensive Cancer Center, David Geffen School of Medicine at UCLA, Los Angeles,
}

Correspondence should be addressed to Timothy F. Lane, tlane@mednet.ucla.edu

Received 3 April 2010; Accepted 7 June 2010

Academic Editor: Ashis Basu

Copyright () 2010 Susan A. Krum et al. This is an open access article distributed under the Creative Commons Attribution License, which permits unrestricted use, distribution, and reproduction in any medium, provided the original work is properly cited.

\begin{abstract}
Following genotoxic stress, the histone H2AX becomes phosphorylated at serine 139 by the ATM/ATR family of kinases. The tumor suppressor BRCA1, also phosphorylated by ATM/ATR kinases, is one of several proteins that colocalize with phospho-H2AX ( $\gamma$ $\mathrm{H} 2 \mathrm{AX}$ ) at sites of active DNA repair. Both the precise mechanism and the purpose of BRCA1 recruitment to sites of DNA damage are unknown. Here we show that BRCA1 and $\gamma$-H2AX form an acid-stable biochemical complex on chromatin after DNA damage. Maximal association of BRCA1 with $\gamma$-H2AX correlates with reduced global $\gamma$-H2AX levels on chromatin late in the repair process. Since BRCA1 is known to have E3 ubiquitin ligase activity in vitro, we examined H2AX for evidence of ubiquitination. We found that $\mathrm{H} 2 \mathrm{AX}$ is ubiquitinated at lysines 119 and 119 in vivo and that blockage of $26 \mathrm{~S}$ proteasome function stabilizes $\gamma$-H2AX levels within cells. When BRCA1 levels were reduced, ubiquitination of H2AX was also reduced, and the cells retained higher levels of phosphorylated H2AX. These results indicate that BRCA1 is recruited into stable complexes with $\gamma$-H2AX and that the complex is involved in attenuation of the $\gamma$-H2AX repair signal after DNA damage.
\end{abstract}

\section{Introduction}

One of the first observable responses to DNA damage is activation of DNA-PK family kinases and resulting phosphorylation of the histone variant $\mathrm{H} 2 \mathrm{AX}$ on $\mathrm{S}_{139}$ [1]. Due to the availability of excellent antibodies to $S_{139}$-phosphorylated $\mathrm{H} 2 \mathrm{AX}$ ( $\mathrm{a}$ form of the protein called $\gamma$-H2AX), this modification is a widely recognized early marker of both genotoxic stress and normal DNA replication [2]. The tail region of H2AX includes a conserved SQ motif $\left(\mathrm{S}_{139} \mathrm{Q}_{140}\right)$ that is recognized as the core target motif of DNA-PK family serine/threonine kinases (ATM [3], ATR [4], and DNA-PK $[3,5])$.

Within minutes after DNA damage, $\gamma$-H2AX becomes identifiable and is localized to discrete nuclear foci [6]. The foci actually include large areas of chromatin flanking points of DNA damage [6]. After DNA damage, several proteins are recruited to regions of $\gamma-\mathrm{H} 2 \mathrm{AX}$ staining. These include the breast cancer susceptibility gene BRCA1, RAD51 [7], the NBS1/RAD50/MRE11 complex [1,8], 53BP1 [9, $10]$, and MDC1 [11, 12]. Recruitment of most proteins to radiation-induced foci is dependent on ATM/ATR activity and formation of $\gamma-\mathrm{H} 2 \mathrm{AX}$, indicating that H2AX phosphorylation plays a key role in maintenance of irradiationinduced foci [13]. ATM is the major H2AX kinase in response to $\gamma$-irradiation [3] while ATR plays a larger role during DNA synthesis [4]. $S_{139}$ phosphorylation of $\mathrm{H} 2 \mathrm{AX}$ is greatly reduced in ATM/ATR knockout cells and is completely blocked by treatment with wortmannin [3], an inhibitor of DNA-PK kinases.

Genetic and biochemical experiments support roles for BRCA1 in homologous recombination [7, 14], nonhomologous end joining $[15,16]$, and transcription-coupled repair [17]. BRCA1 null cells are extremely sensitive to $\gamma$-irradiation 
and other types of genotoxic stress $[18,19]$. Although the role of BRCA1 in DNA repair is not known, the N-terminal ring finger of BRCA1 interacts with the ring finger of BARD1 [20], and the complex has been shown to possess E3 ubiquitin ligase activity in vitro [21]. The E2 ubiquitinconjugating enzyme (UbcH5c) has been shown to associate with this complex $[21,22]$, and several in vitro substrates have been identified, including monoubiquitinated histones $\mathrm{H} 2 \mathrm{AX}, \mathrm{H} 2 \mathrm{~A}, \mathrm{H} 2 \mathrm{~B}, \mathrm{H} 3$, and $\mathrm{H} 4$ (but not H1) [22]. So far, in vivo targets of the complex have been less clearly defined.

BRCA1 association with chromatin is properly considered an intermediate or late event in chromatin repair $[1,23]$. Here we demonstrate by differential fractionation of chromatin bound BRCA1 complexes that BRCA1 and $\gamma$-H2AX form a biochemical complex in the chromatin fraction of cells as a late event following DNA damage. The complex is resistant to nonionic detergent extraction and is dependent on wortmannin-sensitive kinases, features that are distinct from BRCA1 prior to genomic stress. We show that a phosphomimetic of $\mathrm{H} 2 \mathrm{AX}\left(\mathrm{H} 2 \mathrm{AX}-\mathrm{E}_{139}\right)$ is ubiquitinated in vivo, and the major site of ubiquitination is on $\mathrm{K}_{118}$ and/or $\mathrm{K}_{119}$. When BRCA1 levels were reduced using an antisense morpholino knockdown strategy, we observed substantially reduction in $\mathrm{H} 2 \mathrm{AX}$ ubiquitination and increased amounts of $\mathrm{H} 2 \mathrm{AX} \mathrm{S}_{139}$ phosphorylation. These results are consistent with the hypothesis that BRCA1 is present in non-chromatin-associated complexes (including processive RNA polymerase II) prior to genotoxic stress; it becomes phosphorylated, moves into a stable chromatin-associated complex containing $\gamma$-H2AX, and then targets $\gamma$-H2AX for turnover as a late phase of DNA repair.

\section{Materials and Methods}

2.1. Plasmids. $\mathrm{H} 2 \mathrm{AX}, \mathrm{H} 2 \mathrm{AX}-\mathrm{A}_{139}$, and $\mathrm{H} 2 \mathrm{AX}-\mathrm{E}_{139}$, were generated by RT-PCR from human RNA using primers, described in the supplemental data (see Table 1 in Supplementary Material available online at doi: 10.4061/2011/ 801594), and cloned into pcDNA3.1D-V5-H6 (Invitrogen) to generate epitope-tagged variants. PCR-mediated mutagenesis was performed [24] using primers described in the supplementary data to generate additional mutations. pMTHA-Ub (hemagglutinin tagged- ubiquitin) was the generous gift of Dr. Dirk Bohmann (University of Rochester).

2.2. Cell Culture. 293T, MCF-7, and HBL100 were maintained as described in [25].

2.3. DNA Damage and Wortmannin. Damage-randomly cycling HBL100 cells were treated with $4 \mu \mathrm{M}$ adriamycin or were exposed to $10 \mathrm{~Gy}$ ionizing radiation using a ${ }^{137} \mathrm{Cs}$ source (Mark 1 irradiator, Shepherd and Associates). Following treatments, cells were returned to a $5 \% \mathrm{CO}_{2}$ incubator for the indicated amount of time. Cells treated with wortmannin were first pretreated with $100 \mu \mathrm{M}$ wortmannin for $15 \mathrm{~min}$ utes [3].
2.4. Immunoprecipitations and Immunoblotting. Nuclei were prepared by extracting cells in EBC buffer $(50 \mathrm{mM}$ Tris $\mathrm{pH}$ 8.0, $120 \mathrm{mM} \mathrm{NaCl}$, and $0.5 \% \mathrm{NP}-40$ ) as described in [25]. Nuclei were then treated with $0.1 \mathrm{M} \mathrm{HCl}$ for 30 minutes and neutralized with $0.1 \mathrm{M} \mathrm{NaOH}$. This acid soluble "chromatin fraction" was centrifuged for 5 minutes at 14,000 RPM to remove insoluble material. Lysates were precleared with protein $A$ or $G$ beads and then incubated overnight with primary antibodies to $\gamma$-H2AX (Upstate Biotechnology) or BRCA1 (Ab-4, EMD Biosciences). Extracts were run on a $5 \%$ or $14 \%$ SDS-PAGE gel. Proteins were then transferred to nitrocellulose membranes, blocked in 5\% nonfat dry milk, and incubated with a primary antibody generated against BRCA1 (Ab-4, EMD Biosciences), $\gamma$-H2AX (Upstate Biotechnology), H2A (H-124, Santa Cruz Biotechnology), RNA polymerase II (N-20, Santa Cruz Biotechnology or 8WG16 (provided by Dr. Michael Carey (UCLA)), FCP1 (provided by Dr. Michael E. Dahmus (UC Davis)), or $\beta$-actin (Sigma), followed by a goat antirabbit (or antimouse) horseradish peroxidase-conjugated secondary antibody (Pierce). Blots were developed with a chemiluminescence detection substrate (SuperSignal, Pierce).

2.5. Immunocytochemistry. Cells were fixed in $4 \%$ paraformaldehyde (PFA) for 30 minutes on ice. $\gamma$-H2AX was detected with an antibody from Upstate Biotechnology. Alexa-488-conjugated secondary antibodies (Molecular Probes) were used to visualize immune complexes, and photomicrographs were prepared as described in [25]. Quantitation of fluorescent $\gamma$-H2AX foci was accomplished using a Laser Scanning Cytometer (LSC, CompuCyte). Potential autofluorescence was carefully gated by tuning the lasers to optimize signal.

2.6. Proteasome and Phosphatase Inhibitors. Human MCF-7 or $293 \mathrm{~T}$ cells were treated with $5 \mu \mathrm{M}$ adriamycin for 1 hour. Following adriamycin treatment, the cells were washed then treated with or without lactacystin (Sigma) or a serine/threonine phosphatase inhibitor cocktail [(-)p-Bromotetramisole Oxalate, Cantharidin and MicrocystinLR] (EMD Biosciences) for 4 hours, after which the cells were fixed in $4 \%$ PFA. $\gamma$-H2AX was immunostained as described above. Quantitation of fluorescent $\gamma$-H2AX foci was accomplished using a Laser Scanning Cytometer (LSC, CompuCyte).

2.7. Antisense Morpholino Oligos. Two antisense morpholino oligos (Gene Tools, Inc.) were designed against BRCA1 (AS1 5' -GCGAAGAGCAGATAAATCCATTTCT-3' and AS2 5' TGTGCTGACTTACCAGATGGGACAC-3' ). $20 \mu \mathrm{L}$ of each of the two $500 \mu \mathrm{M}$ morpholino antisense oligos were delivered into the cells using the EPEI delivery solution according to the manufacturer's protocol. The remaining cells were treated with a serum-free media control, EPEI delivery solution (Gene Tools, Inc.), or $40 \mu \mathrm{L}$ of a $500 \mu \mathrm{M}$ scrambled control oligo (5'-CCTCTTACCTCAGTTACAATTTATA$\left.3^{\prime}\right)$. After a two-hour treatment, the media were replaced with serum-containing media. The cells were allowed to 


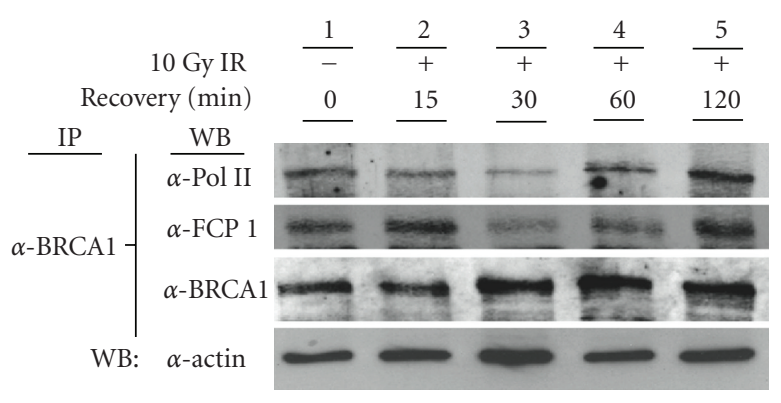

(a) Soluble (RNA pol II) BRCA1 complexes

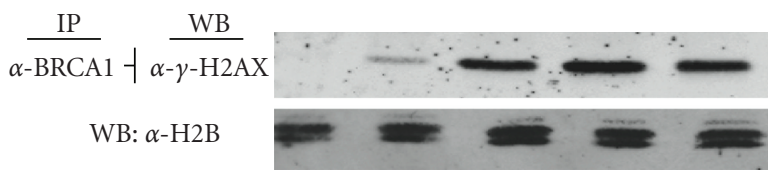

(b) Chromatin $(\gamma-\mathrm{H} 2 \mathrm{AX})$ associated BRCA1

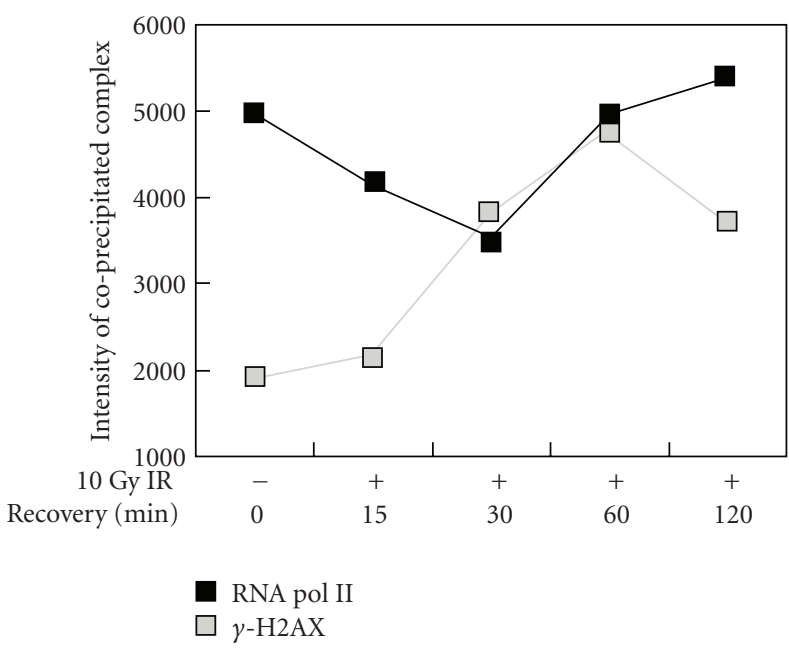

(c) Soluble versus chromatin bound to BRCA1
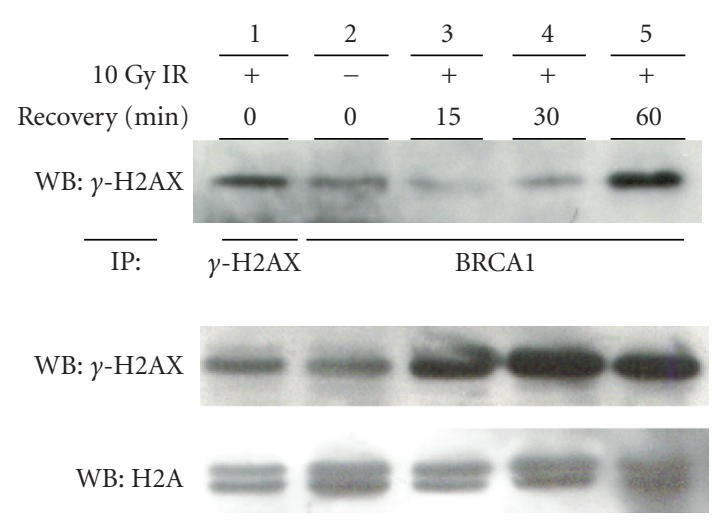

(d) BRCA1: $\gamma$-H2AX complexes coincide with decreased cellular $\gamma$-H2AX

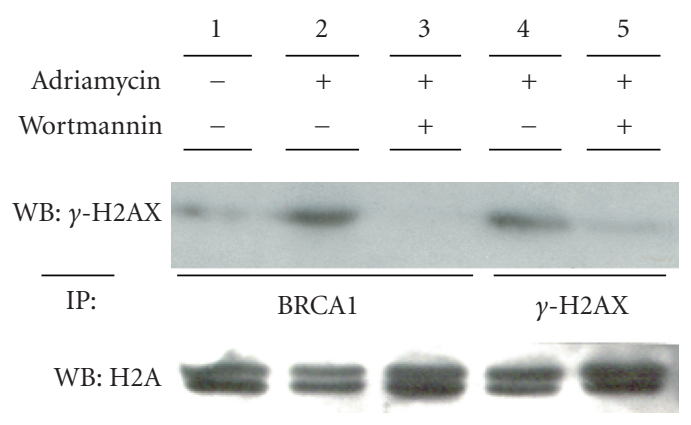

(e) Sensitivity to ATM/ATR inhibitors

FIGURE 1: BRCA1 moves into a chromatin complex containing $\mathrm{S}_{139}$ phosphorylated H2AX ( $\gamma$-H2AX) after DNA damage in vivo. HBL100 cells were exposed to $10 \mathrm{~Gy} \gamma$-irradiation (IR) or $4 \mu \mathrm{M}$ adriamycin then allowed to recover for the indicated time before isolation of protein complexes. (a) Nonchromatin (soluble) nuclear proteins from panel (a) were immunoprecipitated (IP) with antisera to BRCA1. Immunoprecipitates were treated with phosphatase (CIP) and immunoblotted (WB) using antibodies to RNA polymerase II (Pol II, 8WG16), FCP1, or BRCA1. 10\% of the lysate was immunoblotted directly and probed for $\beta$-actin. (b) Total $\gamma$-H2AX and BRCA1-associated $\gamma$-H2AX were immunoprecipitated from the chromatin fraction of cells following IR. $10 \%$ of the chromatin fraction was blotted directly and probed for total H2B. (c) BRCA1 complexes identified in (a) and (b) were quantified and graphed as a function of time. (d) Chromatinassociated protein complexes were extracted (chromatin fraction) of cells exposed to IR as indicated. BRCA1 was immunoprecipitated (IP) from $90 \%$ of the chromatin extract, and immunoblots (WB) were probed with an antibody to $\gamma-\mathrm{H} 2 \mathrm{AX}$ ( $\mathrm{S}_{139}$ phosphorylated $\left.\mathrm{H} 2 \mathrm{AX}\right) .10 \%$ of the chromatin fraction was blotted directly and probed for total H2A. (e) Cells were pretreated with wortmannin for 15 minutes prior to treatment with $4 \mu \mathrm{M}$ adriamycin for $1 \mathrm{hr}$. $90 \%$ of the chromatin fraction was immunoprecipitated (IP) with antibodies to either BRCA1 or $\gamma-\mathrm{H} 2 \mathrm{AX}$ and blotted (WB) with an antibody to $\gamma-\mathrm{H} 2 \mathrm{AX}$. $10 \%$ of the chromatin fraction was blotted directly and probed for total $\mathrm{H} 2 \mathrm{~A}$.

recover for 24 hours at which time they were lysed with EBC buffer. Data related to dose and effectiveness are provided (see supplementary data)

\section{Results}

3.1. BRCA1 Interacts with $\gamma-H 2 A X$. BRCA1 and $\gamma$-H2AX form a physical complex on chromatin following DNA damage. Before DNA damage, small amounts of BRCA1 are present in the chromatin fraction, consistent with the percentage of S-phase nuclei in this population of cells $[4,7,26]$. After treatment with the DNA-damaging agent adriamycin, there is a substantial increase in acid-stable nuclear BRCA1: $\gamma$-H2AX complexes (Figure 1(a)). Stable interaction is minimal at early times following DNA damage then increases to the maximum at about 60 minutes. The 
association is blocked by the ATM/ATR inhibitor wortmannin (Figure 1(b)). This data supports the hypothesis that interaction of BRCA1 and H2AX occurs well after DNA damage, requires phosphorylation by ATM or ATR $[3,27]$, and provides evidence that the interaction requires the formation of a stable complex.

3.2. Time Course of the BRCA1 Complex Formation. In order to analyze the kinetics of BRCA1 interactions, we examined BRCA1 complexes present in undamaged cells and in cells responding to DNA damage. In undamaged cells, we had previously demonstrated that BRCA1 interacts with the phosphorylated, transcriptionally active form of RNA polymerase II (RNA pol II) [25]. Following $\gamma$-irradiation, we found that the BRCA1: $\gamma$-H2AX complex was enhanced (Figure 1(a)) whereas the BRCA1:RNA pol II interaction is disrupted (Figure 1(c)). This shift accompanies the movement of BRCA1 from an easily extractable form in undamaged cells to a chromatin-associated form in damaged cells. The association between BRCA1 and $\gamma$-H2AX peaks about 30-60 minutes after DNA damage whereas the association between BRCA1 and RNA polymerase II is at the lowest 30-60 minutes after DNA damage (Figure 1(d)). FCP1, a protein that is part of the elongating pol II complex, has a similar pattern of association with BRCA1 as that of pol II (Figure 1(c)), illustrating the reciprocal functionality between BRCA1: $\gamma$-H2AX complex and the BRCA1:RNA pol II complex.

Further analysis of the timing of the BRCA1: $\gamma$-H2AX complex shows that this complex coincides with a decreased level of cellular $\gamma$-H2AX. When the BRCA1: $\gamma$-H2AX complex is at its highest level (about 60 minutes after DNA damage), the level of $\gamma$-H2AX has begun to decrease, as demonstrated by immunoblot of the total chromatin extract (Figure 1(e)).

3.3. H2AX Is Ubiquitinated In Vivo. Because BRCA1 is an E3 ubiquitin ligase, we hypothesized that the reduction of $\gamma$-H2AX seen following association with BRCA1 could be the result of ubiquitin-mediated proteasomal degradation. If this were true, then proteasome inhibitors should result in stabilized levels of $\gamma$-H2AX. BRCA1 has been shown to ubiquitinate $\mathrm{H} 2 \mathrm{AX}$ in vitro [22]; therefore, we wanted to determine if $\gamma$-H2AX turnover was proteasome mediated. To test whether $\gamma$-H2AX was degraded by the $26 \mathrm{~S}$ proteasome, MCF-7 mammary epithelial cells were treated with the DNAdamaging agent adriamycin then treated with or without the proteasome inhibitor lactacystin for an additional hour before fixation. Cells were fixed and immunostained to identify $\gamma$-H2AX high and $\gamma$-H2AX low cells. As shown in Figure 2(a), lactacystin increased the percentage of $\gamma$ $\mathrm{H} 2 \mathrm{AX}$ high cells in the population of cells recovering from adriamycin treatment.

By this $\gamma$-H2AX immunofluorescence assay, 20\% of untreated cells were found to be positive for high levels of $\gamma$-H2AX (Figure 2(b)). When cells were treated with adriamycin for 1 hour followed by 1 hour recovery, $\gamma$ H2AX immunofluorescence showed a 2-fold increase over control cells, consistent with the view that H2AX was dynamically phosphorylated in response to DNA damage. The proteasome inhibitor lactacystin had a similar effect as adriamycin, when added alone, consistent with the hypothesis that $\gamma$-H2AX formation in normal cycling cells could be stabilized by proteasome inhibitors. When adriamycin-induced damage was followed by lactacystin treatment, there was a fourfold increase in the amount of $\gamma$-H2AX over control, resulting in over $80 \%$ of cells containing high levels of $\gamma$-H2AX by two hours. Addition of a phosphatase inhibitor cocktail also stabilized $\gamma$-H2AX level, suggesting that there may be additional ways to attenuate H2AX phosphorylation in cells. However, the phosphatase inhibitors alone did not cause stabilization or activation of $\gamma$-H2AX, indicating that proteasome-mediated turnover was a general mechanism to be addressed in further detail. These results provide evidence that $\gamma$ - $\mathrm{H} 2 \mathrm{AX}$ is degraded by the proteasome and potentially by other mechanisms requiring dephosphorylation of one or more components after DNA damage.

3.4. H2AX Is Ubiquitinated at Lysine 118 and/or 119. Previous reports have shown that histone $\mathrm{H} 2 \mathrm{~A}$ is polyubiquitinated on $\mathrm{K}_{119}$, and $\mathrm{H} 2 \mathrm{~B}$ had shown evidence of ubiquitination on $\mathrm{K}_{120}$ [28]. To examine the role of ubiquitin modifications in $\gamma$-H2AX turnover we created a series of modifications intended to model phosphorylation at $S_{139}$ and the roles of various lysine $(\mathrm{K})$ residues in the $\mathrm{C}$ terminus (Figure 3(a) and supplementary Figure 1). H2AX-V5-H6 was first mutagenized to replace $S_{139}$ with alanine (A) or glutamic acid (E), to mimic the negative charge of phosphorylation at $\mathrm{S}_{139}$. As predicted, $\mathrm{H} 2 \mathrm{AX}-\mathrm{S}_{139}-\mathrm{V} 5-\mathrm{H} 6$ localized to chromatin but was moderately less stable than the wild-type H2AX-V5H6 or $\mathrm{A}_{139}$ mutation products (not shown). Cotransfection of $\mathrm{H} 2 \mathrm{AX}-\mathrm{S}_{139}-\mathrm{V} 5-\mathrm{H} 6$ with a cytosolic form of BRCA1 lacking a nuclear localization sequence (BRCA1- $\Delta 11-\mathrm{GFP}$ ) resulted in nuclear localization of both proteins and rapid turnover (not shown). For these reasons, we hypothesized that $\mathrm{H} 2 \mathrm{AX}-\mathrm{S}_{139}$ was mimicking $\mathrm{S}_{139}$ phosphorylation and recruiting binding of BRCA1.

We then replaced each lysine $(\mathrm{K})$ residue found in the conserved C-terminus of $\mathrm{H} 2 \mathrm{AX}-\mathrm{E}_{139}$ with arginine (R) and tested for ubiquitination in vivo (Figure 3 ). $\mathrm{K}_{118}$ and $\mathrm{K}_{119}$ (which align with $\mathrm{K}_{119}$ and $\mathrm{K}_{120}$ of $\mathrm{H} 2 \mathrm{~A}$ ) were mutated individually (Figure 3(c), lanes 6 and 7) and in combination (lane 8 ). $\mathrm{K}_{133}$ and $\mathrm{K}_{134}$ were also mutated individually (Figure 3(c), lanes 9 and 10) and in combination (lane 11) to arginine. Mutation of $\mathrm{K}_{128}$ to $\mathrm{R}_{128}$ was also prepared as part of this series but had no effect (data not shown). $293 \mathrm{~T}$ cells were transfected with wild-type or mutagenized pcDNA3-H2AX-V5-H6 in addition to HAtagged ubiquitin. H6-tagged complexes were purified using nickel-chelated beads, and bound proteins were analyzed by immunoblotting for the presence of HA-tagged H2AX (Ub-H2AX). As shown, V5 immunoblotting identified modified and ubiquitinated H2AX only when extracts from cotransfected cells were purified and blotted (Figure 3(b)). Reprobing these blots with anti-HA antisera revealed that the upper band contained ubiquitin (Figure $3(\mathrm{~b})$ ). While the 

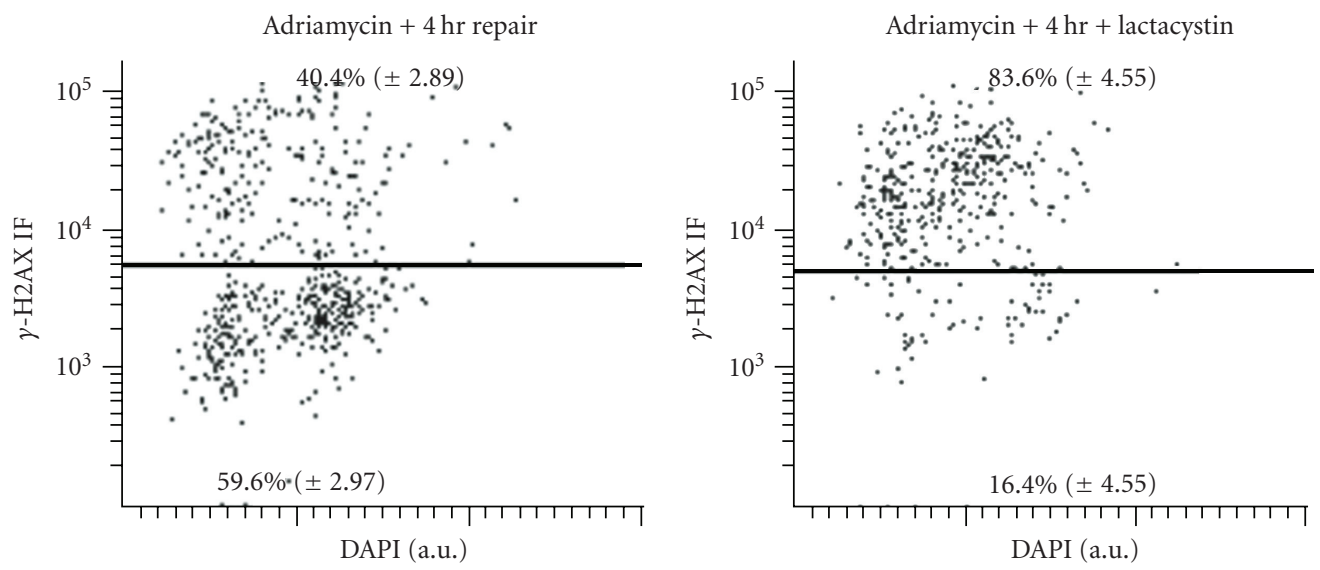

(a) Proteasome inhibitors

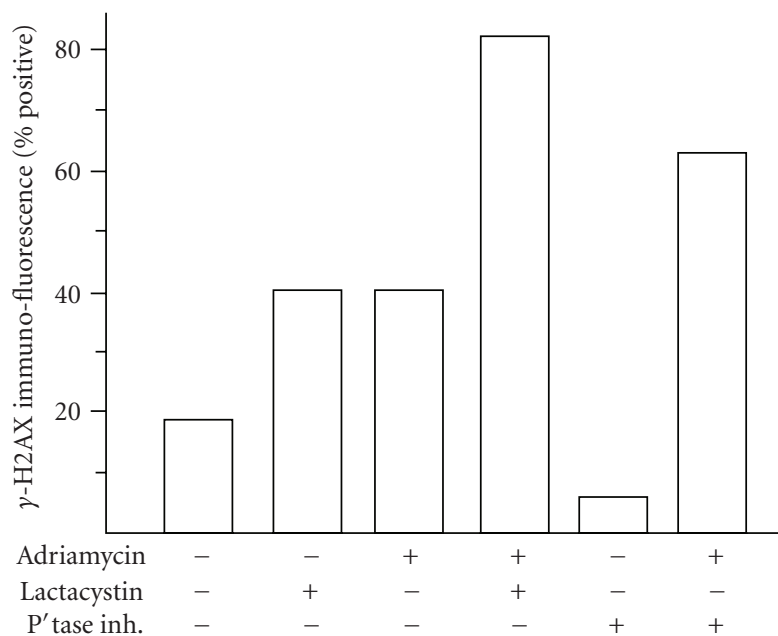

(b) Stability of $\gamma-\mathrm{H} 2 \mathrm{AX}$

FIGURE 2: H2AX is dephosphorylated following DNA damage. MCF-7 cells were treated with adriamycin for 1 hour then treated with lactacystin or a phosphatase inhibitor (P'tase inh.) cocktail, as indicated. Cells were fixed and stained for $\gamma$-H2AX and quantitated using a Laser Scanning Cytometer.

major band migrated in a fashion consistent with that of monoubiquitinated $\mathrm{H} 2 \mathrm{AX}$, there were additional bands of immunoreactivity at higher molecular weights that suggested polyubiquitination as well (Figure 3(b) arrowheads). H2AX mutated at both $\mathrm{K}_{118}$ and $\mathrm{K}_{119}$ showed no ubiquitination (lane 8), indicating that $\mathrm{H} 2 \mathrm{AX}$ is ubiquitinated at either $\mathrm{K}_{118}$ or $K_{119}$, whereas other point mutations showed levels of ubiquitination similar to wild type. These results show that $\mathrm{H} 2 \mathrm{AX}-\mathrm{E}_{139}$ is ubiquitinated at either $\mathrm{K}_{118}$ or $\mathrm{K}_{119}$.

3.5. BRCA1 Knockdown Increases Steady State Levels of $\gamma$ H2AX Buy Reduction of Ubiquitination in Cells Undergoing Replicative Stress. To functionally examine the interaction between BRCA1 and $\gamma$-H2AX, we used antisense morpholino oligonucleotides to knockdown BRCA1 expression. Treatment of cells with BRCA1 antisense oligonucleotides reduced the amount of BRCA1 to under $3 \%$ of normal levels (Figure 4(a), and supplemental data). Reduction of
BRCA1 protein resulted in increased levels of $\gamma$-H2AX expression by both immunofluorescence (Figure 4(a)) and immunoblotting (Figure 4(b)).

Cells treated with antisense oligos directed at BRCA1 mRNA were transfected with H2AX-V5-H6 and HAtagged ubiquitin. The epitope-tagged $\mathrm{H} 2 \mathrm{AX}$ product was then isolated using nickel-chelated beads and analyzed by immunoblotting for the presence of HA-tagged ubiquitin. Cells treated with BRCA1 antisense oligos had a reduction in the global amount of H2AX ubiquitination detected (Figure 4(c)). Efforts to examine the role of double-strand break repair on this process are underway but are complicated by the extreme sensitivity of BRCA1 deficient cells to genotoxic agents.

It has been shown previously that BRCA1 deficiency is associated with G2/M checkpoint and other defects [29-32]. To examine the relationship between BRCA1 and genotoxic stress more carefully, we examined cells treated with BRCA1 


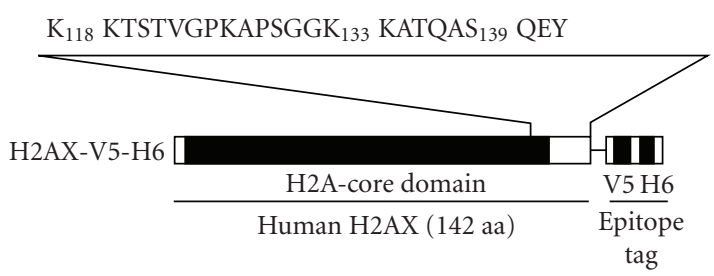

(a) Analysis of H2AX-Ub complexes

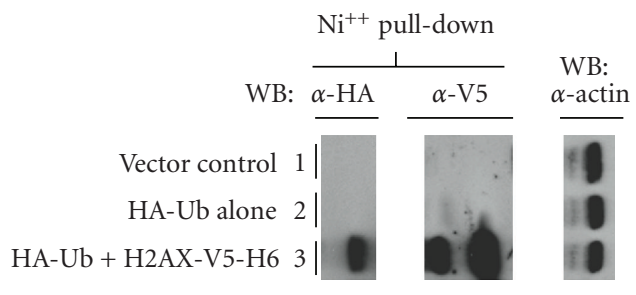

(b) Ubiquitination of H2AX

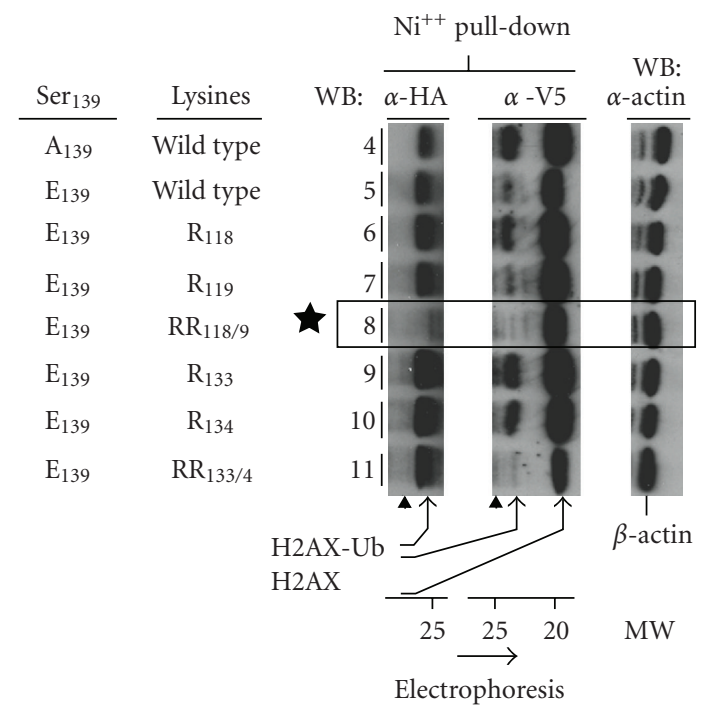

(c) Mutational analysis of H2AX

Figure 3: H2AX-E $\mathrm{E}_{139}$ is ubiquitinated at $\mathrm{K}_{118}$ or $\mathrm{K}_{119}$. (a) Critical amino acids within the C-terminus are shown. (b) HA-tagged ubiquitin (HA-Ub) was transfected into cells with or without H2AX-V5-H6. His-tagged (H6) proteins were purified from chromatin fractions then probed sequentially for the presence of HAubiquitin and V5 epitopes (WB). 10\% of the unfractionated extract was blotted directly and probed for $\beta$-actin. (c) H2AX variants containing $\mathrm{E}_{139}$ (to mimic $\mathrm{S}_{139}$ phosphorylation) and various lysine (K) to arginine (R) Substitutions were transfected with HA-tagged ubiquitin. Histidine-tagged proteins were purified from chromatin fractions then probed sequentially for the presence of HA-ubiquitin and V5 epitopes. $10 \%$ of the unfractionated extract was blotted directly and probed for $\beta$-actin. The star (lane 8 ) indicates the absence of HA-tagged ubiquitin. Arrows indicate unmodified and ubiquitinated $\mathrm{H} 2 \mathrm{AX}$.

antisense oligos for evidence that $\gamma$-H2AX stabilization correlated with replicative defects. We found that early in the response to BRCA1 knockdown, $\gamma$-H2AX expression was confined to cells in G2/M or late S-phase of the cell cycle (Figure 4(d)). Most cells with 2N DNA, as measured by DAPI fluorescence, had normal $\gamma$-H2AX staining patterns

\section{Discussion}

These results suggest that BRCA1 deficiency is associated with defective clearance of $\gamma$-H2AX from cells following replication and other types of genotoxic stress (Figure 5). We propose that BRCA1 interacts with processive RNA pol II in undamaged cells as part of a role in genomic surveillance (Figure 5(a)) [25, 33]. Following genotoxic stress, phosphorylation of BRCA1 by ATM/ATR and potentially by chk1 results in its dissociation from stalled RNA pol II complexes. We propose that an early repair complex forms on DNA as a consequence of ATM/ATR phosphorylation. Early targets for ATM/ATR include phosphorylation of $\mathrm{H} 2 \mathrm{AX}$ to form $\gamma$-H2AX $[34,35] . \gamma$-H2AX then serves as a template to aid in the recruitment of early and late components of the repair machinery, including 53BP1 (Figure 5(b)) [36]. We propose that BRCA1 is recruited at later times, potentially after break repair has been affected. One target for BRCA1 recruitment is $\gamma$-H2AX, which is directly or indirectly ubiquitinated on $\mathrm{K}_{118}$ or $\mathrm{K}_{119}$ and degraded through the actions of the $26 \mathrm{~S}$ proteasome (Figure $5(\mathrm{c}))$.

We have shown that BRCA1 is in a biochemical complex with $\gamma$-H2AX after DNA damage. This interaction is dependent on the DNA-PK family of kinases (ATM and/or ATR), as the interaction is disrupted by the inhibitor wortmannin. This data agrees with previous data that BRCA1 colocalizes with repair proteins and suggests a function for BRCA1 in the chromatin fraction of nuclei. BRCA1 becomes part of the BRCA1-associated surveillance complex (BASC), which includes many proteins involved in DNA repair, including MSH2, MSH6, MLH1, ATM, BLM, and the RAD50-MRE11-NBS1 protein complex [37]. BRCA1 and the BASC complex are localized at the site of DNA damage (nuclear foci). The repair factors are thus at the site of damage where they can perform their particular enzymatic activities and repair the DNA.

Using a mutant from of $\mathrm{H} 2 \mathrm{AX}\left(\mathrm{H} 2 \mathrm{AX}-\mathrm{E}_{139}\right)$ designed to mimic phosphorylation at $\mathrm{S}_{139}$ in $\gamma$-H2AX, we performed mutagenesis of several conserved lysine residues in the $\mathrm{C}$ terminal end of H2AX. Results from these experiments suggest that ubiquitination is suppressed following mutation of both $\mathrm{K}_{118}$ and $\mathrm{K}_{119}$, but not by mutation of other lysines either alone or in combination. Previously reports also showed that BRCA1 was capable of supporting ubiquitination of $\mathrm{H} 2 \mathrm{AX}$, but those studies were carried out in cell-free reactions in vitro [22]. Ubiquitination of $\mathrm{K}_{118}$ and $\mathrm{K}_{119}$ agrees with tryptic peptide data [38] that showed ubiquitination between residues 118 and 127.

We propose that a major function of BRCA1 is to decrease the levels of $\gamma-\mathrm{H} 2 \mathrm{AX}$ in cells as a mechanism for signaling an end to early events in DNA repair. BRCA1 activity is then critical for timely attenuation of active repair phase. Defective production of BRCA1 in tumor cells would be expected to result in less ordered diminution of the repair signal and lead to problems in progression though $\mathrm{G} 2 / \mathrm{M}$ phases of the cell cycle. 


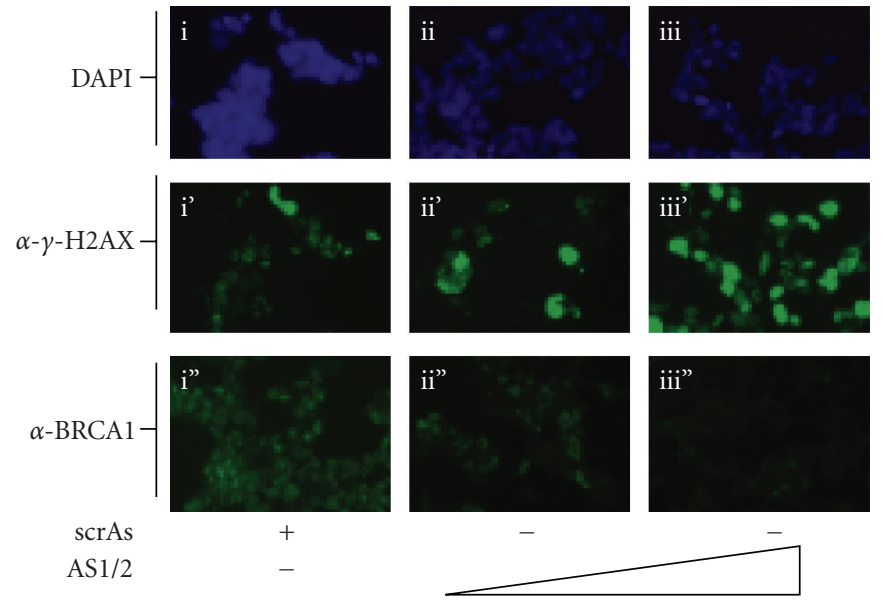

(a) BRCA1 loss stabilizes $\gamma$-H2AX

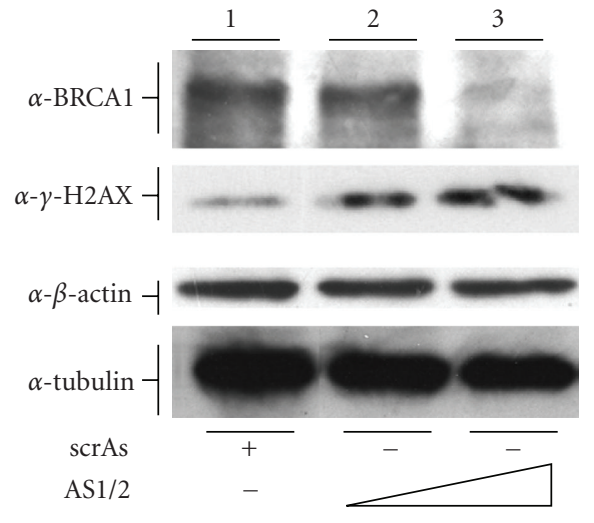

(b)
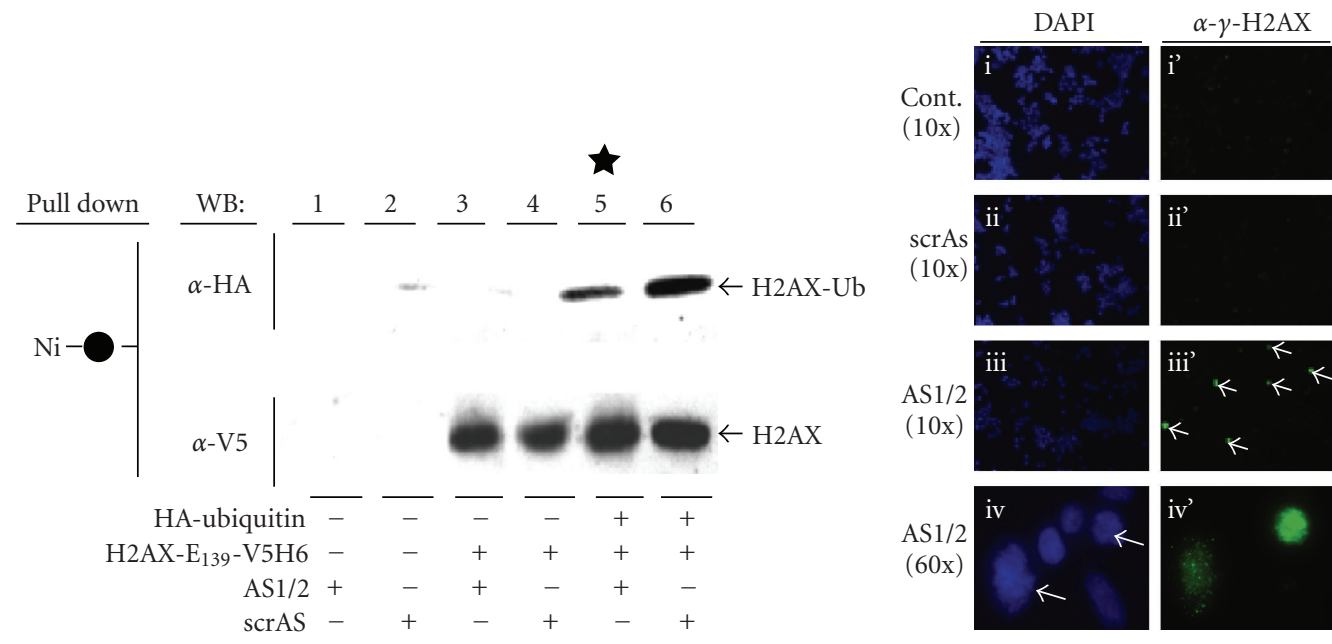

(c) BRCA1 knockdown reduces ubiquitination of $\gamma$-H2AX mimetics
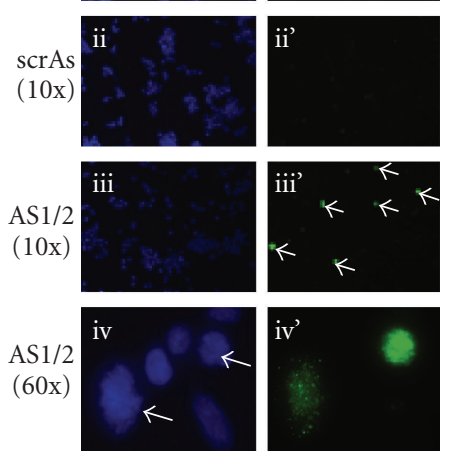

(d) $\gamma$-H2AX increases in G2/M

FIGURE 4: BRCA1 knockdown stabilizes $\gamma$-H2AX and reduces ubiquitination of $\gamma$-H2AX. (a) Cells treated with different amounts of antisense morpholino oligos (AS1 and AS2) or a scrambled antisense morpholino (scrAS) were stained with DAPI or immunostained for $\gamma$-H2AX. A second set of morpholino-treated cells was immunostained for BRCA1 to show dose-dependent knockdown of BRCA1 protein. (b) Cells treated with scrambled antisense morpholino (lane 1, scrAS) or increasing amounts of antisense morpholino oligos (AS1/2, lanes 2 and 3 ) were lysed and separated by SDS-PAGE and probed (WB) for BRCA1, $\gamma$-H2AX, $\beta$-actin, or $\beta$-tubulin. (c) $293 \mathrm{~T}$ cells were treated with control (scrambled antisense (scrAS) or BRCA1 antisense morpholino oligos (AS1/2) and then transfected with control vector, HA-ubiquitin alone, and/or H2AX-E139-V5-H6. Histidine- (H6-) tagged H2AX was purified from chromatin fractions then probed for HA-ubiquitin. The blot was stripped and reprobed for V5-tag on H2AX. (d) Cells treated with a scrambled antisense morpholino (scrAS) or anti-BRCA1 morpholino oligos (AS1/2) were stained with DAPI (i-iv) or immunostained for $\gamma$-H2AX (i'-iv'). Micrographs were captured with either 10x (i-iii) or 60x (iv) objectives. Final magnification was 100x or 600x.

\section{Acknowledgments}

The authors are grateful for an NRSA predoctoral fellowship (no. GM07185) to Susan A. Krum. Esther de la Rosa Dalugdugan was supported by the UCLA CARE Program and by a Howard Hughes Medical Institute grant to the Undergraduate Biological Sciences Education Program of UCLA. Initial funding came from the UCLA Human Gene
Medicine Program, and the Stop Cancer Foundation, to Timothy F. Lane. The project also received support from the Ovarian Cancer Research Fund, the DOD C.D.M.R.P Breast Cancer Initiative, and the Cancer Research Coordinating Committee of the University of California. The authors thank Robert Lurvey for assistance with mutagenesis of H2AX and members of the Lane Lab for many helpful discussions. 


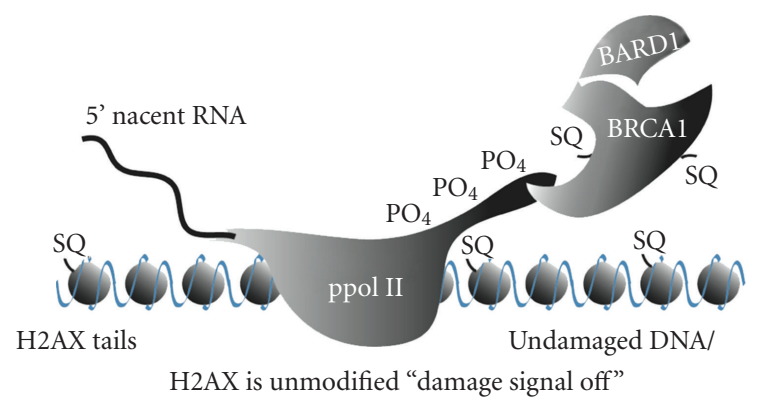

(a) BRCA1: pRNApol II complex in undamaged cells

ATM/ATR-dependent DNA damage repair machinery recruited by phosphorylation of $\mathrm{H} 2 \mathrm{AX}$ at position $\mathrm{S}_{139}$

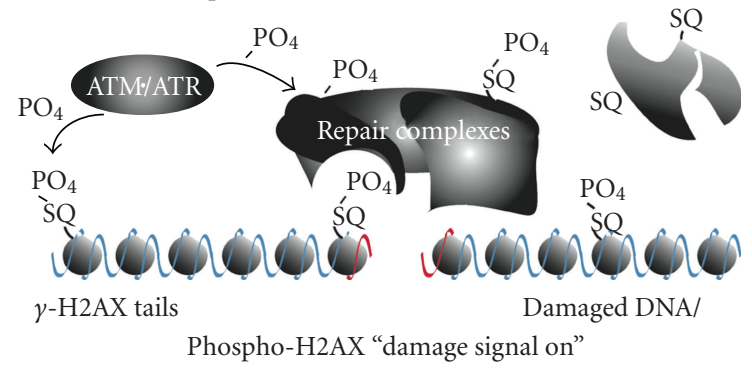

(b) Early repair complexes with $\gamma$-H2AX lack BRCA1

ATM/ATR-dependent recruitment of BRCA1 results in turnover of $\gamma$-H2AX

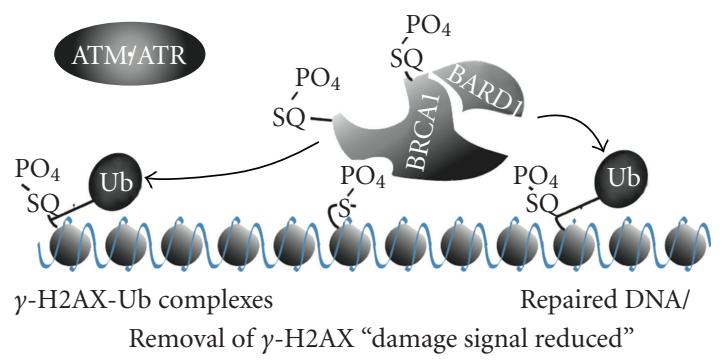

(c) Late repair complexes with $\gamma-\mathrm{H} 2 \mathrm{AX}$ and BRCA1

Figure 5: Model of BRCA1 interactions with RNA polymerase II and $\gamma$-H2AX in distinct biochemical complexes. See text for details.

\section{References}

[1] T. T. Paull, E. P. Rogakou, V. Yamazaki, C. U. Kirchgessner, M. Gellert, and W. M. Bonner, "A critical role for histone $\mathrm{H} 2 \mathrm{AX}$ in recruitment of repair factors to nuclear foci after DNA damage," Current Biology, vol. 10, no. 15, pp. 886-895, 2000.

[2] E. P. Rogakou, D. R. Pilch, A. H. Orr, V. S. Ivanova, and W. M. Bonner, "DNA double-stranded breaks induce histone H2AX phosphorylation on serine 139," The Journal of Biological Chemistry, vol. 273, no. 10, pp. 5858-5868, 1998.

[3] S. Burma, B. P. Chen, M. Murphy, A. Kurimasa, and D. J. Chen, "ATM phosphorylates histone H2AX in response to DNA double-strand breaks," The Journal of Biological Chemistry, vol. 276, no. 45, pp. 42462-42467, 2001.

[4] I. M. Ward and J. Chen, "Histone H2AX is phosphorylated in an ATR-dependent manner in response to replicational stress," The Journal of Biological Chemistry, vol. 276, no. 51, pp. 47759-47762, 2001.

[5] E.-J. Park, D. W. Chan, J.-H. Park, M. A. Oettinger, and J. Kwon, "DNA-PK is activated by nucleosomes and phosphorylates $\mathrm{H} 2 \mathrm{AX}$ within the nucleosomes in an acetylationdependent manner," Nucleic Acids Research, vol. 31, no. 23, pp. 6819-6827, 2003.

[6] E. P. Rogakou, C. Boon, C. Redon, and W. M. Bonner, "Megabase chromatin domains involved in DNA doublestrand breaks in vivo," Journal of Cell Biology, vol. 146, no. 5, pp. 905-915, 1999.

[7] R. Scully, J. Chen, A. Plug et al., "Association of BRCA1 with Rad51 in mitotic and meiotic cells," Cell, vol. 88, no. 2, pp. 265-275, 1997.

[8] T. Furuta, H. Takemura, Z.-Y. Liao et al., "Phosphorylation of histone $\mathrm{H} 2 \mathrm{AX}$ and activation of Mre11, Rad50, and Nbs1 in response to replication-dependent DNA double-strand breaks induced by mammalian DNA topoisomerase I cleavage complexes," The Journal of Biological Chemistry, vol. 278, no. 22, pp. 20303-20312, 2003.

[9] S. Fan, J.-A. Wang, R. Yuan et al., "BRCA1 inhibition of estrogen receptor signaling in transfected cells," Science, vol. 284, no. 5418, pp. 1354-1356, 1999.

[10] O. Fernandez-Capetillo, S. K. Mahadevaiah, A. Celeste et al., "H2AX is required for chromatin remodeling and inactivation of sex chromosomes in male mouse meiosis," Developmental Cell, vol. 4, no. 4, pp. 497-508, 2003.

[11] G. S. Stewart, B. Wang, C. R. Bigneli, A. M. R. Taylor, and S. J. Elledge, "MDC1 is a mediator of the mammalian DNA damage checkpoint," Nature, vol. 421, no. 6926, pp. 961-966, 2003.

[12] A. Peng and P.-L. Chen, "NFBD1, like 53BP1, is an early and redundant transducer mediating Chk2 phosphorylation in response to DNA damage," The Journal of Biological Chemistry, vol. 278, no. 11, pp. 8873-8876, 2003.

[13] A. Celeste, O. Fernandez-Capetillo, M. J. Kruhlak et al., "Histone H2AX phosphorylation is dispensable for the initial recognition of DNA breaks," Nature Cell Biology, vol. 5, no. 7, pp. 675-679, 2003.

[14] M. E. Moynahan, J. W. Chiu, B. H. Koller, and M. Jasint, "Brcal controls homology-directed DNA repair," Molecular Cell, vol. 4, no. 4, pp. 511-518, 1999.

[15] H. Wang, Z.-C. Zeng, T.-A. Bui et al., "Nonhomologous endjoining of ionizing radiation-induced DNA double-stranded breaks in human tumor cells deficient in BRCA1 or BRCA2," Cancer Research, vol. 61, no. 1, pp. 270-277, 2001.

[16] C. Baldeyron, E. Jacquemin, J. Smith et al., "A single mutated BRCA1 allele leads to impaired fidelity of double strand break end-joining," Oncogene, vol. 21, no. 9, pp. 1401-1410, 2002.

[17] L. C. Gowen, A. V. Avrutskaya, A. M. Latour, B. H. Koller, and S. A. Leadon, "BRCA1 required for transcription-coupled repair of oxidative DNA damage," Science, vol. 281, no. 5379, pp. 1009-1012, 1998.

[18] N. Chiba and J. D. Parvin, "Redistribution of BRCA1 among four different protein complexes following replication blockage," The Journal of Biological Chemistry, vol. 276, no. 42, pp. 38549-38554, 2001.

[19] R. Scully, J. Chen, R. L. Ochs et al., "Dynamic changes of BRCA1 subnuclear location and phosphorylation state are initiated by DNA damage," Cell, vol. 90, no. 3, pp. 425-435, 1997.

[20] L. C. Wu, Z. W. Wang, J. T. Tsan et al., "Identification of a RING protein that can interact in vivo with the BRCA1 gene product," Nature Genetics, vol. 14, no. 4, pp. 430-440, 1996. 
[21] R. Hashizume, M. Fukuda, I. Maeda et al., "The RING heterodimer BRCA1-BARD1 is a ubiquitin ligase inactivated by a breast cancer-derived mutation," The Journal of Biological Chemistry, vol. 276, no. 18, pp. 14537-14540, 2001.

[22] D. L. Mallery, C. J. Vandenberg, and K. Hiom, "Activation of the E3 ligase function of the BRCA1/BARD1 complex by polyubiquitin chains," The EMBO Journal, vol. 21, no. 24, pp. 6755-6762, 2002.

[23] A. Rothfuss and M. Grompe, "Repair kinetics of genomic interstrand DNA cross-links: evidence for DNA double-strand break-dependent activation of the Fanconi anemia/BRCA pathway," Molecular and Cellular Biology, vol. 24, no. 1, pp. 123-134, 2004.

[24] M. Carey and S. T. Smale, Transcriptional Regulation in Eukaryotes: Concepts, Strategies, and Technique, Cold Spring Harbor Laboratory, 2001.

[25] S. A. Krum, G. A. Miranda, C. Lin, and T. F. Lane, "BRCA1 associates with processive RNA polymerase II," The Journal of Biological Chemistry, vol. 278, no. 52, pp. 52012-52020, 2003.

[26] Y. Chen, A. A. Farmer, C.-F. Chen, D. C. Jones, P.-L. Chen, and W.-H. Lee, "BRCA1 is a $220-\mathrm{kDa}$ nuclear phosphoprotein that is expressed and phosphorylated in a cell cycle-dependent manner," Cancer Research, vol. 56, no. 14, pp. 3168-3172, 1996.

[27] D. Cortez, Y. Wang, J. Qin, and S. J. Elledge, "Requirement of ATM-dependent phosphorylation of Brcal in the DNA damage response to double-strand breaks," Science, vol. 286, no. 5442, pp. 1162-1166, 1999.

[28] L. J. M. Jason, S. C. Moore, J. D. Lewis, G. Lindsey, and J. Ausió, "Histone ubiquitination: a tagging tail unfolds?" BioEssays, vol. 24, no. 2, pp. 166-174, 2002.

[29] R.-H. Wang, H. Yu, and C.-X. Deng, "A requirement for breast-cancer-associated gene 1 (BRCA1) in the spindle checkpoint," Proceedings of the National Academy of Sciences of the United States of America, vol. 101, no. 49, pp. 17108-17113, 2004.

[30] X. Wang, R.-H. Wang, W. Li et al., "Genetic interactions between Brcal and Gadd45a in centrosome duplication, genetic stability, and neural tube closure," The Journal of Biological Chemistry, vol. 279, no. 28, pp. 29606-29614, 2004.

[31] M. Ouchi, N. Fujiuchi, K. Sasai et al., "BRCA1 phosphorylation by Aurora-A in the regulation of $\mathrm{G}_{2}$ to $\mathrm{M}$ transition," The Journal of Biological Chemistry, vol. 279, no. 19, pp. 1964319648, 2004.

[32] L. M. Starita, Y. Machida, S. Sankaran et al., "BRCA1dependent ubiquitination of $\gamma$-tubulin regulates centrosome number," Molecular and Cellular Biology, vol. 24, no. 19, pp. 8457-8466, 2004.

[33] P. Čabart, H. K. Chew, and S. Murphy, "BRCA1 cooperates with NUFIP and P-TEFb to activate transcription by RNA polymerase II," Oncogene, vol. 23, no. 31, pp. 5316-5329, 2004.

[34] P. J. McKinnon, "ATM and ataxia telangiectasia. Second in molecular medicine review series," EMBO Reports, vol. 5, no. 8, pp. 772-776, 2004.

[35] N. Motoyama and K. Naka, "DNA damage tumor suppressor genes and genomic instability," Current Opinion in Genetics and Development, vol. 14, no. 1, pp. 11-16, 2004.

[36] B. Wang, S. Matsuoka, P. B. Carpenter, and S. J. Elledge, "53BP1, a mediator of the DNA damage checkpoint," Science, vol. 298, no. 5597, pp. 1435-1438, 2002.

[37] Y. Wang, D. Cortez, P. Yazdi, N. Neff, S. J. Elledge, and J. Qin, "BASC, a super complex of BRCA1-associated proteins involved in the recognition and repair of aberrant DNA structures," Genes and Development, vol. 14, no. 8, pp. 927939, 2000.

[38] M. H. P. West, R. S. Wu, and W. M. Bonner, "Polyacrylamide gel electrophoresis of small peptides," Electrophoresis, vol. 5, no. 3, pp. 133-138, 1984. 

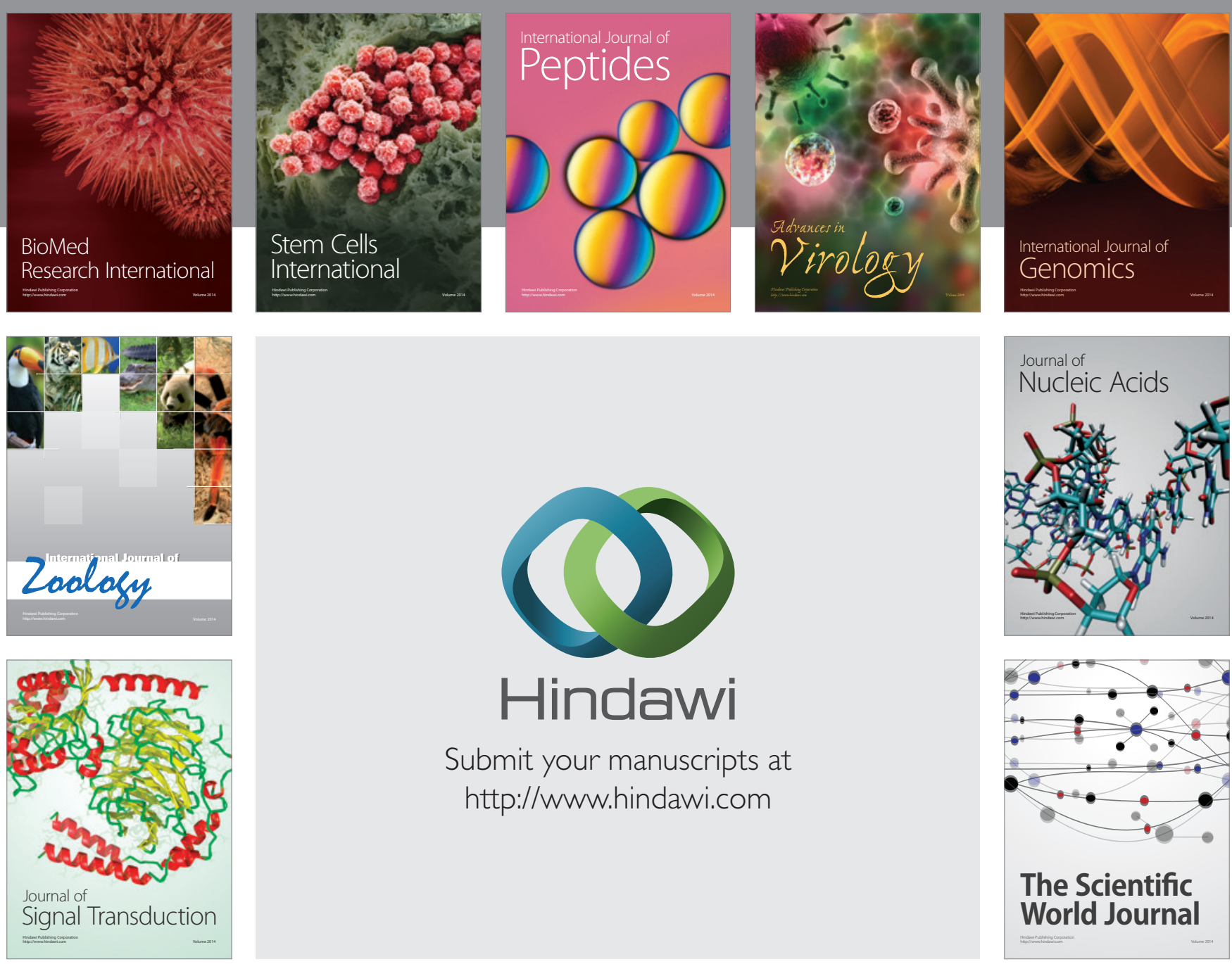

Submit your manuscripts at

http://www.hindawi.com
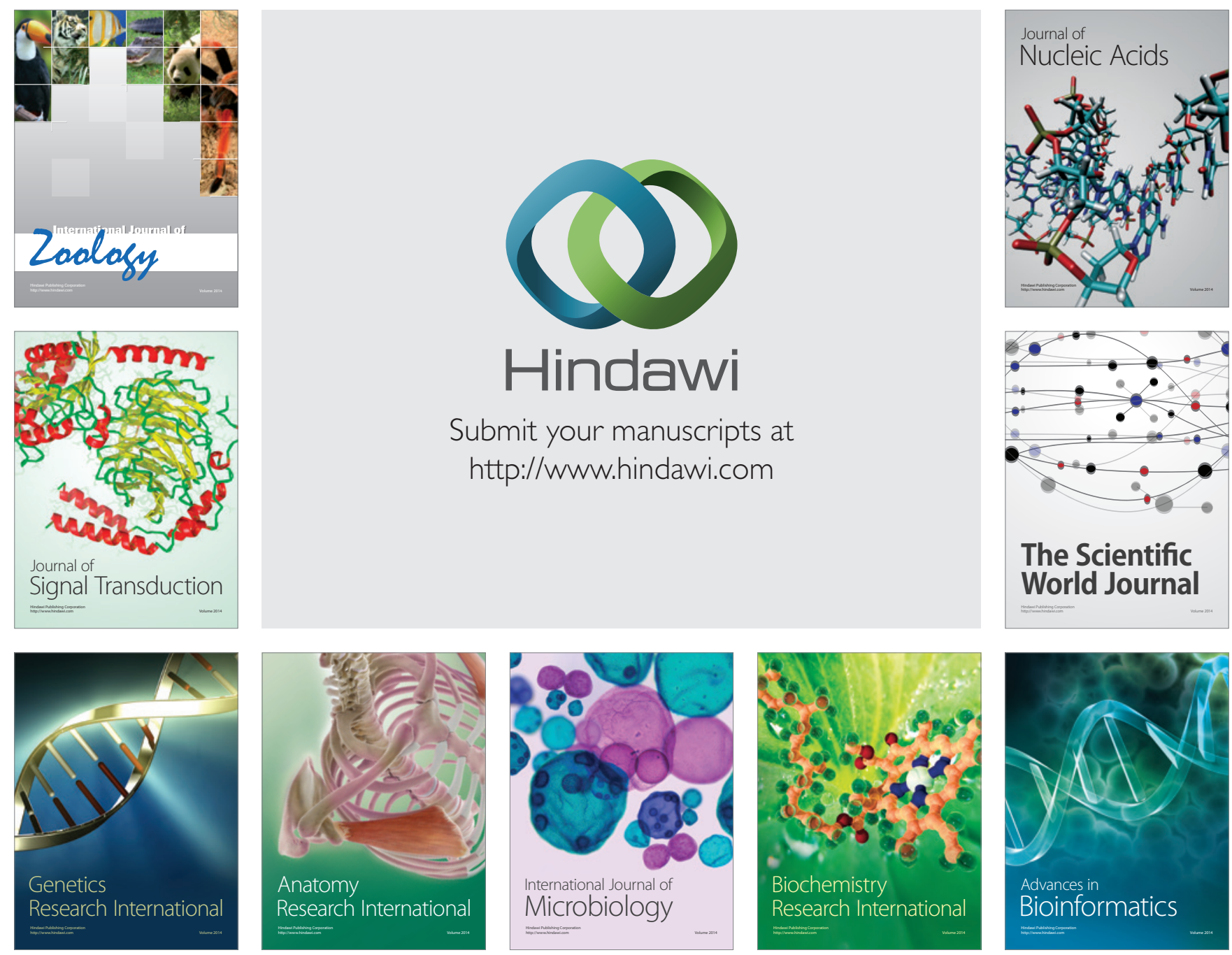

The Scientific World Journal
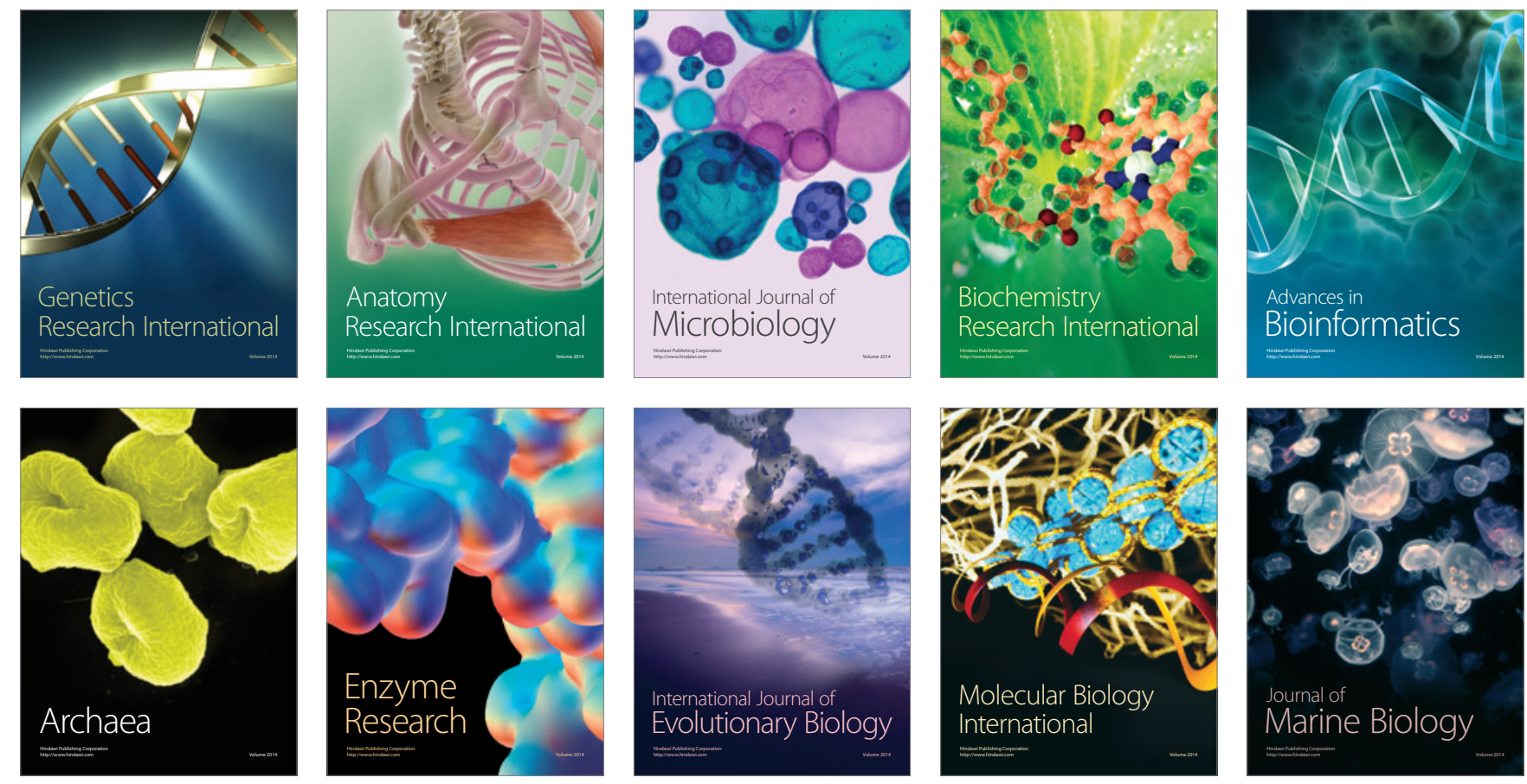